\title{
Enfuvirtide-Induced Cutaneous Amyloidosis
}

\author{
Lohini Sundharkrishnan, MD; David M. Pilkington, MD; Jeffrey P. North, MD
}

\section{PRACTICE POINTS}

- There are multiple types of cutaneous amyloidosis, and proper diagnosis is essential to direct treatment and follow-up care.

- Medication-associated amyloidosis is a rare type of amyloidosis that is not associated with systemic amyloidosis and is treated by switching to alternative medicines.

\section{To the Editor:}

Cutaneous amyloidosis can be secondary to many causes. We describe a case of amyloidosis that was secondary to the deposition of an antiretroviral drug enfuvirtide and clinically presented as bullae over the anterior abdominal wall.

A 65-year-old man with HIV presented with pink vesicles and flaccid bullae on the anterolateral aspect of the lower abdomen (Figure 1) in areas of self-administered subcutaneous injections of enfuvirtide. He reported tissue swelling with a yellow discoloration immediately after injections that would spontaneously subside after a few minutes.

A biopsy from the left lateral abdomen revealed dilated vessels concentrically encompassed by pink globular material and nodular collections of the pink amorphous substance in the upper dermis (Figure 2), which was accompanied by a sparse, perivascular, lymphohistiocytic inflammatory infiltrate; scattered plasma cells; and rare eosinophils in a background of dermal edema. Although Congo red stain was negative, crystal violet revealed metachromatic staining of the globular material that was highlighted as dark violet against a blue background. Given these clinical and histopathologic findings, a diagnosis of drug-induced amyloidosis was made.
Amyloidosis refers to a group of disorders that result from misfolding of proteins in the characteristic betapleated sheet structure that can accumulate in various tissues. There are different subtypes of amyloidosis based on the type of protein deposited: immunoglobulin light chain protein (AL); serum amyloid A (AA), an acute-phase reactant accumulating in those with longstanding inflammatory conditions; beta-2 microglobulin (Ab2M) in patients with renal failure; keratin in macular and lichen amyloidosis; pharmaceutical-derived amyloid (eg, enfuvirtide, injectable insulin); and mutated proteins in hereditary amyloidosis such as transthyretin. ${ }^{1}$ Other familial forms include genetic variants of apolipolipoprotein AII (AApoAI, AApoAII), fibrinogen A alpha chain (AFib), lysozyme (ALys), cystatin C (ACys), and gelsolin (AGel). ${ }^{2}$

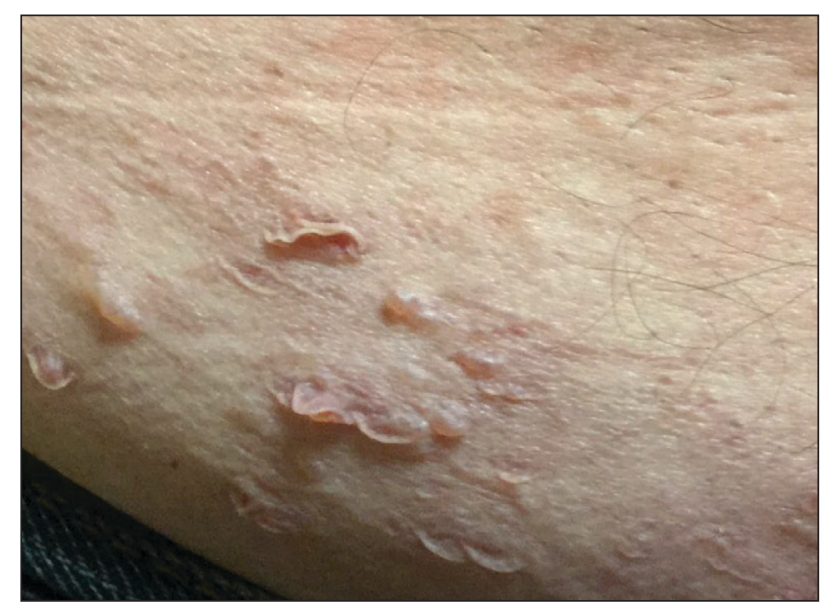

FIGURE 1. Cutaneous amyloidosis. Flesh-colored vesicles and flaccid bullae scattered over the abdomen.

Dr. Sundharkrishnan is from Dermpath Diagnostics, Newtown Square, Pennsylvania. Dr. Pilkington is from Pilkington Dermatology, Merced, California. Dr. North is from the University of California San Francisco. 


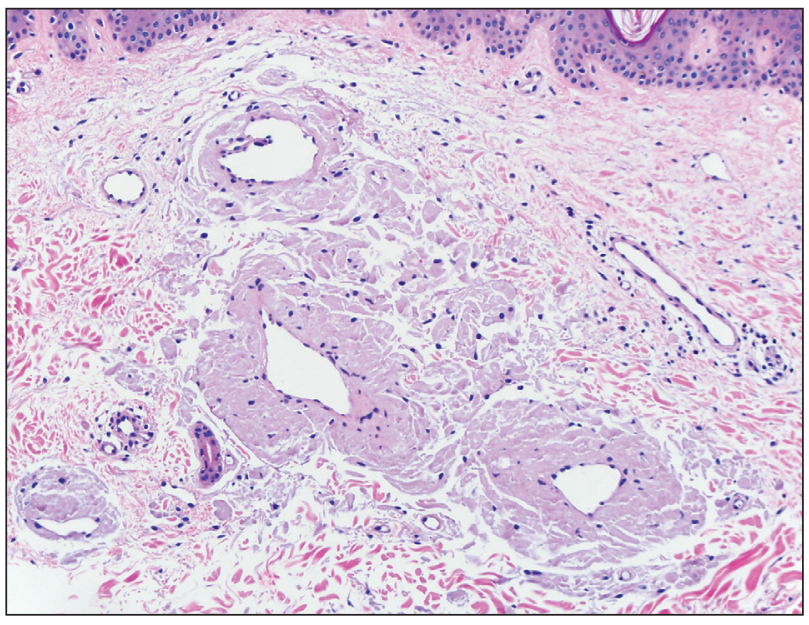

FIGURE 2. Hyalinized, pink, globular material consistent with amyloid surrounding blood vessels in the upper dermis (H\&E, original magnification $\times 200$ ).

Cutaneous amyloidosis can stem from a systemic disease or arise as a localized phenomenon. Primary cutaneous amyloidosis can present as either macular, lichen, or nodular forms. The pathogenesis of cutaneous nodular amyloidosis differs from that of lichen and macular types and results from deposition of light chain-derived amyloid protein. In contrast, lichen and macular subtypes have keratin-derived amyloid deposits in the papillary dermis and stain positive for keratin antibodies, especially cytokeratins 5 and 6 . Primary nodular amyloidosis has a $7 \%$ to $50 \%$ risk for developing systemic amyloidosis and a $9 \%$ risk for local recurrence, hence the necessity to assess for monoclonal gammopathy with urine light chains and serum immunoelectrophoresis. ${ }^{3}$

Drug-induced amyloidosis is a distinct type of cutaneous amyloidosis that histopathologically resembles nodular amyloidosis. Multiple drugs have been reported in this setting: insulin,, 5 enfuvirtide injections, and liraglutide. ${ }^{6}$ Enfuvirtide belongs to a class of antiretroviral agents and is a synthetic peptide composed of 36 amino acids. It inhibits the fusion of HIV with the host helper T cell by binding to glycoprotein $41 .^{7}$ Enfuvirtide-related amyloidosis was described in 3 case reports, 2 that confirmed enfuvirtide as the amyloid constituent by protein analysis. ${ }^{8-10}$ One study analyzed the amyloid proteome in 50 cases of insulin-derived amyloidosis and 2 cases of enfuvirtide-derived amyloidosis. Laser microdissection-tandem microscopy revealed that the amyloid in such cases was composed of the drug enfuvirtide itself along with deposits of apolipoproteins (E, A-I, A-IV) and serum amyloid $\mathrm{P}$ component. ${ }^{4}$ Additional complications can occur at the site of enfuvirtide injections. A retrospective review of 7 patients with injection-site reactions to enfuvirtide described erythema, induration, and nodules, with histopathologic findings including hypersensitivity reactions and palisaded granulomas resembling granuloma annulare. Amorphous material was noted within histiocytes and in the surrounding connective tissue that was confirmed as enfuvirtide by immunoperoxidase staining. ${ }^{11}$

In summary, several types of cutaneous amyloidosis occur, including secondary cutaneous involvement by systemic amyloidosis and drug-induced amyloidosis, and notable histopathologic overlap exists between these types. Given the differing treatment requirements depending on the type of cutaneous amyloidosis, obtaining an appropriate clinical history, including the patient's medication list, is important to ensure the correct diagnosis is reached. Protein analysis with mass spectrometry can be used if the nature of the amyloid remains indeterminate.

\section{REFERENCES}

1. Merlini G, Bellotti V. Molecular mechanisms of amyloidosis. N Engl J Med. 2003;349:583-596.

2. Ferri FF. Amyloidosis. In: Ferri F. Ferri's Clinical Advisor 2016: 5 Books in 1. Elsevier; 2016.

3. Kaltoft B, Schmidt G, Lauritzen AF, et al. Primary localised cutaneous amyloidosis—a systematic review. Dan Med J. 2013;60:A4727.

4. D'Souza A, Theis JD, Vrana JA, et al. Pharmaceutical amyloidosis associated with subcutaneous insulin and enfuvirtide administration. Amyloid. 2014;21:71-75.

5. Sie MP, van der Wiel HE, Smedts FM, et al. Human recombinant insulin and amyloidosis: an unexpected association. Neth J Med. 2010;68:138-140.

6. Martins CO, Lezcano C, Yi SS, et al. Novel iatrogenic amyloidosis caused by peptide drug liraglutide: a clinical mimic of AL amyloidosis. Haematologica. 2018;103:E610-E612.

7. Lazzarin A, Clotet B, Cooper D, et al. Efficacy of enfuvirtide in patients infected with drug-resistant HIV-1 in Europe and Australia. $N$ Engl J Med. 2003;348:2186-2195.

8. Naujokas A, Vidal CI, Mercer SE, et al. A novel form of amyloid deposited at the site of enfuvirtide injection. J Cutan Pathol. 2012; 39:220-221; quiz 219.

9. Mercer S, Whang T, Vidal C, et al. Massive amyloidosis at the site of enfuvirtide (Fuzeon) injection. J Cutan Pathol. 2011;38:93.

10. Morilla ME, Kocher J, Harmaty M. Localized amyloidosis at the site of enfuvirtide injection. Ann Intern Med. 2009;151:515-516.

11. Ball RA, Kinchelow T; ISR Substudy Group. Injection site reactions with the HIV-1 fusion inhibitor enfuvirtide. J Am Acad Dermatol. 2003;49:826-831. 